\title{
Cardiovascular System Findings Numeric Result in Standard Unit
}

National Cancer Institute

\section{Source}

National Cancer Institute. Cardiovascular System Findings Numeric Result in Standard

Unit. NCI Thesaurus. Code C124017.

The numerical identifier of a cardiovascular system finding result in standard units. 